\title{
Sistematización de la experiencia Reconocimiento de los derechos humanos del adulto mayor en dos familias residentes en Cali y Valledupar (Colombia)
}

\author{
Lina María Cuello-Lacouture \\ Magíster en Asesoría Familiar. Psicóloga \\ Banco de Occidente. Valledupar, Colombia \\ https://orcid.org/0000-0003-3328-2940 • lcuello@bancodeoccidente.com.co \\ Jimena del Pilar Jaramillo-Jaramillo \\ Magíster en Asesoría Familiar. Trabajadora Social \\ Universidad Antonio José Camacho. Cali, Colombia \\ https://orcid.org/0000-0003-0010-2616• jimenajaramillo@opendeusto.es
}

Resumen

El presente artículo contiene los resultados de la Sistematización de la Experiencia del Proyecto de Asesoría Familiar: "Reconocimiento de los derechos humanos del Adulto Mayor en dos familias residentes en Cali y Valledupar". Se desarrolló durante los meses de febrero a noviembre del año 2017, a través de un proceso participativo con las familias S. P. en Valledupar y C. V. en Cali, para alcanzar el reconocimiento familiar y social de los adultos mayores como sujeto de derechos, fomentando su participación y realzando sus recursos y competencias, desde una perspectiva de derechos humanos y del apoyo intergeneracional.

Ahora bien, la construcción del proyecto se realizó por medio de la integración de aportes de la Investigación Acción Participativa y la Metodología del Marco Lógico; la implementación fue ejecutada en escenarios de asesoría familiar desde un Enfoque Sistémico en el que emergieron dos ejes fundamentales: la participación social y la corresponsabilidad. Finalmente, en los resultados fue predominante el importante papel que ejerce la familia para que los adultos mayores sean reconocidos como sujetos de derecho.

Palabras clave: Adultez mayor; Derechos humanos; Relaciones intergeneracionales; Participación social; Corresponsabilidad.

Recibido: 13/10/2020 | Aprobado: 14/11/2020 |Publicado: 01/01/2021

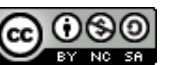

Esta obra está bajo una Licencia Creative Commons Atribución-NoComercialCompartirIgual 4.0 Internacional.

Financiación o proveniencia del artículo: artículo derivado de la Sistematización de la Experiencia del Proyecto de Asesoría Familiar: "Reconocimiento de los derechos humanos del Adulto Mayor en dos familias residentes en Cali y Valledupar, desarrollada entre febrero y noviembre de 2017 en Valledupar y Cali, Colombia.

¿Cómo citar este artículo? / How to quote this article?

Cuello-Lacouture, L. M, y Jaramillo-Jaramillo, J. d. P. (2021). Sistematización de la experiencia Reconocimiento de los derechos humanos del adulto mayor en dos familias residentes en Cali y Valledupar (Colombia). Prospectiva. Revista de Trabajo Social e intervención social, (31), 201-219. doi: $10.25100 /$ prts.v0i31.10565. 
Cuello-Lacouture y Jaramillo-Jaramillo

\title{
Systematization of Experience Recognition of the Human Rights of Elderly Citizens in Two Families Residing in Cali and Valledupar (Colombia)
}

\begin{abstract}
The systematization of the experience of the Family Counseling Project: "Recognition of the human rights of the elderly in two families residing in Cali and Valledupar"; was developed during the months of February to November 2017, through a participatory process with the families S.P. in Valledupar and C.V. in Cali. The aim was to achieve family and social recognition of the elderly as subjects with rights, encouraging their participation and enhancing their resources and skills, from a human rights and intergenerational support perspective.

The construction of the project was carried out through the integration of contributions from Participatory Action Research and the Logical Framework Methodology; the implementation was executed in family counseling scenarios from a Systemic Approach in which two fundamental axes emerged: social participation and co-responsibility. Finally, in the results, the important role played by the family in the recognition of the elderly as subjects of law was predominant.

Keywords: Elderly adulthood; Human rights; Intergenerational relations; Social participation; Co-responsibility.

Sumario: 1. Introducción, 2. Objeto de la sistematización, 3. Metodología, 4. Hallazgos, 4.1 Re-creación compartida de la experiencia y co-construcción de sentidos, 4.2 Descripción y análisis de la experiencia desde la perspectiva de la asesoría familiar, 5. Conclusiones, 6. Referencias Bibliográficas.
\end{abstract}




\section{Introducción}

La Sistematización de la experiencia del proyecto "Reconocimiento de los derechos humanos del adulto mayor en dos familias residentes en Cali y Valledupar" se realizó para contribuir a la transformación familiar y social de las familias S. P. y C. V. que transitan la etapa del ciclo vital adultez mayor.

En las últimas décadas en Colombia, han emergido una serie de cambios sociodemográficos que inciden en el incremento del porcentaje de población que supera los sesenta años y la esperanza de vida media, por ejemplo, en el año 1974 fue de 62 años y en el 2016 ascendió a 76 años de edad, lo cual ha acelerado la transición demográfica que según el Departamento Administrativo Nacional de Estadística-DANE (2017), se convierte en un desafío para el sector público quien debe ejecutar políticas públicas orientadas al desarrollo, la calidad de vida y el cumplimiento de los derechos humanos para la inclusión social de los adultos mayores.

Una de las problemáticas sociales actuales está relacionada con la baja calidad de vida de esta población y el desconocimiento de sus derechos, situación que obstaculiza su evolución como lo esbozan Bazo y García (2006). Se encuentra que los adultos mayores experimentan condiciones particularmente difíciles, ya que en el imaginario de la sociedad occidental como plantea Elías (2009) la población es valorada desde el plano fisiológico, por lo tanto, los adultos mayores tienden a ser excluidos y a su vez, no representan una opción efectiva y rentable para el desarrollo de actividades laborales y sociales.

De tal manera, el presente proyecto de asesoría familiar tuvo como objetivo, en dos familias colombianas, fortalecer el reconocimiento familiar y social del Adulto Mayor como sujeto de derechos, fomentando la participación y realzando sus recursos y competencias, desde una perspectiva de derechos humanos y del apoyo intergeneracional, lo que se constituye en una fuente de reflexión y aporte en la generación de nuevos proyectos para la atención del adulto mayor.

La etapa de formulación e implementación se desarrolló con la metodología de la Investigación Acción Participativa (IAP) como herramienta transversal durante todo el proceso y, contribuyó a la generación de espacios para que los adultos mayores fueran los protagonistas, se sensibilizaran de sus propios problemas, profundizaran en el análisis y propusieran alternativas autónomas para el bien común. Además, se utilizó la metodología del Marco Lógico con base en los planteamientos de Cotera-Fretel (2012) y se procedió a construir el árbol de problemas, seguido por el árbol de objetivos, presupuesto, cronograma, actividades, indicadores, resultados esperados y mecanismos de evaluación. 
Cuello-Lacouture y Jaramillo-Jaramillo

Con respecto a la Sistematización de la experiencia, el método fue planificado a partir de los elementos conceptuales propuestos por Alboan, Hegoa y Universidad de Deusto (2004), Ayllón (2001) y Barnechea y Morgan (2007). Se consideraron cinco momentos: formulación, recopilación de información inicial, diseño y aplicación de instrumentos, interpretación y análisis de la información obtenida. La metodología que se utilizó fue el medio para recuperar la información de fuentes primarias y los principales elementos teóricos y prácticos que giraron alrededor de la experiencia vivida, con el propósito de suscitar aprendizajes que pudieran ser acogidos como punto de referencia desde otros escenarios de construcción y ejecución.

Es así como desde la asesoría familiar surge la necesidad de regenerar el estado homeostático de los sistemas familiares donde los protagonistas fueron los adultos mayores, lo que condujo a considerar el enfoque de derechos humanos como eje transversal del proceso. Toda persona, como entidad dinámica bio-psico-social, adquiere de manera automática al nacer, unos derechos que se van consolidando a través de su desarrollo mediante los vínculos con los otros. Esas relaciones fortalecen al ser humano como un sujeto de derechos y lo acompañan durante los distintos ciclos vitales por los que atraviesa.

Con el propósito de contribuir a fortalecer el reconocimiento individual, familiar y social del Adulto Mayor como sujeto de derechos, se desarrolló el proyecto de asesoría familiar y se sistematizó la experiencia, de la cual se deriva el presente artículo, con la intención de aportar en los cambios sobre esta realidad y producir conocimiento desde las vivencias de estas dos familias, estimulando la transformación de sus realidades mediante procesos colectivos de aprendizaje que refuerzan la intervención vinculada a la asesoría familiar.

\section{Objeto de la sistematización}

En el Proyecto de Asesoría Familiar titulado "Reconocimiento de los derechos humanos del adulto mayor en dos familias de Cali y Valledupar", las familias seleccionadas presentan una característica especial: en ambas existe la presencia de una pareja de adultos mayores de edades superiores a los 65 años, quienes junto a sus demás familiares decidieron compartir sus experiencias, historia de vida e imaginarios sociales sobre la etapa del ciclo vital que transitan: adultez mayor.

Por tal razón, se contrastaron los conceptos sobre la Adultez Mayor con la participación intergeneracional de estas familias, desde una mirada diferencial e intercultural, para propender por su autorreconocimiento en la cotidianidad y la transversalidad de los contextos culturales al ritmo del vallenato y la salsa, entre el folklore de los acentos. 
Cuello-Lacouture y Jaramillo-Jaramillo

Cabe señalar que, el proceso de intervención se realizó desde la asesoría familiar en la que se dimensionaron las familias desde una perspectiva multidimensional, integradora, contextual y reflexiva para construir y resignificar sus experiencias las cuales se moldean en costumbres, comportamientos, actitudes, normas, valores y demás; en ese sentido, la familia constituye un contexto fundamental para la construcción del desarrollo individual, social e intergeneracional. Así mismo, se legitimaron las condiciones relacionales e idiosincráticas de las familias que emergieron durante el desarrollo del proyecto en los escenarios de acción.

En ese orden de ideas, el proceso de asesoría familiar permitió interpretar la diversidad sociocultural de las familias para reconocerlas como sujetos activos portadores de derechos, constructores de ciudadanía, con independencia de género, raza y etnia, con el fin de introducirse en sus experiencias y resignificarlas desde una perspectiva constructora y reconstructora de la realidad.

\section{Metodología}

La metodología Sistematización de experiencias es un tipo de investigación social cualitativa que exige vivir y reconstruir la realidad, incita al dinamismo, la reflexión, la participación y la transformación social mediante la construcción disciplinar y práctica. Por lo tanto, es un proceso integral que compromete una interacción directa entre la comunicación, la socialización y la producción de nuevo conocimiento, como lo esboza Carvajal-Burbano (2006).

Al examinar las implicaciones y desafíos de la Sistematización de experiencias, se encuentra que han sido ampliamente discutidas desde varias posturas científicas. Es así como Torres-Carrillo (1996) declara que esta metodología puede ser vagamente confundida con otros enfoques cualitativos de la investigación social como la Investigación Acción Participativa y la denominada Recuperación Colectiva de la Historia, que se desarrolla simultáneamente, mediante procesos de reconstrucción, interpretación y transformación de la experiencia. En relación con el enfoque, Ghiso (1999) sugiere que a toda sistematización le antecede una práctica y otorga un gran valor al sujeto como ser que percibe la realidad y produce conocimiento. Por esto, se consideró fundamental incorporar los siguientes dos enfoques propuestos por este autor:

Enfoque dialógico e interactivo, según Ghiso (1999) reconoce que la realidad social se construye con base en las interacciones y los diálogos que se gestan en las dos familias como colectivo y de modo individual donde se legitiman el contexto, las voces, las acciones y los referentes externos como marcos teóricos y empíricos acerca de la adultez mayor, sus 
Cuello-Lacouture y Jaramillo-Jaramillo

derechos y alcances, que permiten organizar y coordinar la información particular de éstas, como propuesta del conocimiento unificada.

El enfoque de la reflexividad y la construcción de la experiencia humana, se centra en la observación del contexto de situaciones cotidianas. Para Ghiso (1999) el aprendizaje se produce a través de la reflexión acerca de la experiencia, del diálogo y de la exploración de los significados de acontecimientos en los diferentes contextos de inmersión de las familias. Otorga un valor especial a los juicios y las destrezas de las familias durante los encuentros, con el fin de comprender sus perspectivas y así, aportar a la búsqueda y ejecución de alternativas de solución ante el problema identificado.

Sin desconocer la envergadura de los otros enfoques propuestos por Ghiso (1999) como el hermenéutico, el deconstructivo y el histórico-dialéctico, los dos enfoques seleccionados se aproximaron más a lo que se logró con las dos familias participantes, que buscaron el reconocimiento de sus derechos humanos en la etapa de la adultez mayor.

\subsection{Etapas de la Sistematización}

Se retomó la experiencia y algunos de los aportes metodológicos de Alboan, Hegoa y Universidad de Deusto (2004), Ayllón (2001) y Barnechea y Morgan (2007). A continuación, se enuncian las etapas que contribuyeron en la planeación y la ejecución del proceso de sistematización posterior a la implementación del proyecto de asesoría familiar con las dos familias de adultos mayores que viven en las ciudades de Cali y Valledupar respectivamente.

Formulación, socialización y concertación del Plan de Sistematización y conformación del Grupo de sistematización

- Trabajo individual: en esta primera etapa se procedió a diseñar el Plan de sistematización inicial, junto a la presentación en diapositivas para socializar a las familias. Luego se concertó la cita para la socialización en el lugar de residencia de las familias y también precisiones sobre los botones que iban a llevar, así como el refrigerio que se iba a brindar.

- Técnica - Trabajo grupal e informativo: durante el encuentro de socialización, se mostró la presentación realizada y en cada ítem, se hizo una pausa para preguntar sobre ésta, si adicionarían u omitirían información, registrando cada sugerencia.

- Seguidamente, por medio de una entrevista grupal se abrió un espacio en el que todos escogieron a dos integrantes que contaran con el tiempo y la disposición 
Cuello-Lacouture y Jaramillo-Jaramillo

para reflexionar sobre lo vivido. A los integrantes escogidos se les entregó un botón llamativo que decía "Voy a Sistematizar nuestra experiencia".

- Herramientas: entrevista grupal.

- Insumos: como materiales se llevó un computador con la presentación, hojas, lápices y la Guía Memoria 4. Así mismo, un refrigerio para compartir al finalizar la actividad y los botones que se les entregó a los participantes de la sistematización.

- Una vez socializado, se procedió a perfeccionar el plan de sistematización inicial y a organizar y analizar la información recopilada.

Recopilación de información inicial, fuentes secundarias, identificación de los hitos y los ejes de la Sistematización

- Trabajo individual: partiendo del acumulado del proceso, se realizó una revisión exhaustiva y rigurosa de toda la información existente acerca de la formulación y la implementación del Proyecto y se organizó metódicamente en un cuadro. Lo que corresponde a la etapa de formulación, se llevó a cabo por medio de siete jornadas, cada una de dos horas realizadas entre los meses de febrero a mayo del año 2017. En ese momento, se analizaron los productos de dichas jornadas por cada familia como instrumentos de recolección de información: Informe de conocimiento de la metodología IAP; Selección de las familias por convocar; Planeación general de los encuentros; Construcción del cronograma; Construcción del problema en forma conjunta; En busca del objetivo común y Construcción del proyecto.

- En cuanto a la fase de implementación, ésta se ejecutó de mayo a septiembre del año 2017 a través de doce actividades de dos horas de duración en cada familia.

- Se analizaron los formatos que contenían los registros de los hechos susceptibles de ser interpretados correspondientes a las actividades ejecutadas, así: Formato de actividad 1 "Vamos a empezar"; Formato de actividad 2: "Reconociendo los aspectos biológicos y psicológicos del envejecimiento"; Formato de actividad 3: "Por una cultura de la vejez y el envejecimiento"; Formato de actividad 4: "Reconociendo los aspectos sociales y económicos del envejecimiento"; Formato de actividad 5: "Juegos afectivos para fortalecer los vínculos familiares"; Formato de actividad 6: "Comunicación Asertiva"; Formato de 7: Salida Recreativa; Formato de actividad 8: "Construcción del Folleto Derechos Humanos en 
Cuello-Lacouture y Jaramillo-Jaramillo

Acción"; Formato de actividad 9: "Conversaciones de Externalización"; Formato de actividad 10: "Deconstrucción del relato"; Formato de actividad 11: “Diseñando el ritual" y Formato de actividad 12: "Viviendo el ritual”.

- Técnica - Trabajo grupal e informativo: se realizó la respectiva inspección documental con los participantes tomando como base toda la documentación generada a partir de la formulación e implementación del proyecto, y se seleccionó lo más relevante del proceso llevado a cabo. A su vez, se tuvieron en cuenta los diversos registros de los encuentros como fotografías, vídeos y trabajos de investigación relacionados en los Referentes empíricos.

Posteriormente, se solicitó que establecieran los hitos con base en los momentos más relevantes del proceso para que recordaran las distintas etapas, que fueron:

- Momento 1 - Origen del proyecto. En ambas familias, los adultos mayores señalaron que la vejez y las enfermedades que se presentan en esta etapa de la vida, limitan su participación abierta y flexible en actividades familiares, barriales y en relación con otras redes. De acuerdo con las apreciaciones, se reconoció como hito las dificultades para interactuar con los demás que revelan las personas cuando llegan a esta etapa de la vida, lo que afecta su cotidianeidad y forma de comunicación.

- Momento 2 - Formulación del proyecto. Al revivir este momento, se evocaron las voces de los participantes que mencionaron: “(...) cuando elaboramos el árbol de problemas, nos llevó a reflexionar sobre todos los problemas que existen alrededor de los adultos mayores”, “(...) representó en mí y en el viejo, descargarnos de todo lo que veníamos sintiendo $(\ldots)^{\prime \prime}$, “(...) es que son demasiadas cosas, y lo enfermo y sin ganas que uno se siente (...)” (C.-Valledupar, Septiembre 2017), “( ...) definitivamente, el árbol de problemas fue vital, nos hizo reconocer todo lo que el mundo cree sobre los ancianos, y empoderarnos de las soluciones (...)” (B.-Cali, septiembre 2017), “(...) y también el árbol de objetivos, en el que convertimos los problemas en objetivos y oportunidades para lograr cambios" (R.-Cali, septiembre 2017).

En ese sentido, se definieron como hitos dos actividades fundamentales durante esta etapa que son la identificación del problema y la búsqueda de un objetivo en común. En el árbol de problemas, se consideró como problema "el estigma social del adulto mayor como ser inactivo", para lo cual se determinaron varias causas y efectos, en los que sobresalió la exclusión social, los prejuicios sociales, la improductividad y las redes de apoyo limitadas. Además, las asesoras recordaron 
Cuello-Lacouture y Jaramillo-Jaramillo

que en el Árbol de objetivos quedó plasmado el interés de la familia para que se reconocieran a los adultos mayores como sujetos de derechos.

- Momento 3 - Implementación del proyecto. Cuando se reflexionó sobre esta etapa, ambas familias plantearon dos actividades que se consideraron hitos y son: la salida recreativa y la socialización de la cartilla en el hogar geriátrico, momentos cumbres durante todo el proceso, ya que “(...) nos permitió disfrutar de espacios que normalmente no visitamos, y compartir en familia con usted y ser responsables con los demás viejitos (...)” (R.-Cali, Septiembre 2017), “(...) ir a la casa de los ancianos, nos permitió valorar lo que somos y tenemos, y a comprender que sí somos sujetos de derechos y valiosos para la sociedad, y podemos aportarles $(. .) ”,. ~ “(. .$.$) y bueno, reconozco que realmente si cuidan a los$ abuelos allá (...)" (C.-Valledupar, Septiembre 2017).

- Momento 4 - Resultados del proyecto. Finalmente, al razonar sobre la etapa de resultados, enunciaron que fue un proceso “(...) muy especial, nos permitió vivir y sentir de manera distinta, somos importantes y podemos dar más para mejorar este mundo (...)” (B.-Cali, septiembre 2017), “(...) realmente valoro el que nos haya escogido al viejo y a mí, nos hizo sentir valiosos y ahora mi familia así lo ve (...)" (C.-Valledupar, septiembre 2017). Así mismo, estuvieron a la expectativa de concluir el proceso de la mejor manera, reflexionar sobre lo aprendido y colaborar hasta el final.

Posteriormente y con base en los hallazgos del ejercicio anterior, se identificaron dos ejes en la sistematización que actuaron como hilo conductor durante todo el proceso y son: la participación social de los adultos mayores y la corresponsabilidad comprendida como responsabilidad compartida de manera intergeneracional. Una vez seleccionados los hitos, se pidió que construyeran los ejes de la sistematización, con base en dos etapas claves: la formulación y la implementación del proyecto. Para finalizar, se solicitó que escogieran a los informantes calificados, es decir, aquellas personas que fueron testigos del proceso durante la formulación y la implementación del proyecto.

- Herramientas: Revisión documental y entrevista semiestructurada.

- Insumos: Guía de Memoria 4, en la que se plasmaron los detalles de lo experimentado durante la actividad. Así mismo, se hizo uso de una ficha de registro de datos, con el fin de identificar y organizar los datos bibliográficos y fuentes secundarias seleccionadas. 
Cuello-Lacouture y Jaramillo-Jaramillo

Diseño de instrumentos para la recopilación de información - fuentes primarias

- Trabajo individual: Se diseñaron dos instrumentos para la recopilación de información de fuentes primarias: una entrevista semiestructurada y un debate de grupo. El diseño de estos instrumentos estuvo basado en los ejes de sistematización identificados y en los objetivos de la Sistematización relacionados con cada uno de ellos.

De esta manera, la entrevista grupal semiestructurada se realizó sobre el eje de corresponsabilidad y se construyó un formulario a través de una lista de preguntas establecidas con anterioridad con el fin de "estimular el relato principal del entrevistado" (Flick, 2004, p. 111).

Por su parte, el debate de grupo se construyó alrededor del eje de participación social y fue el instrumento ideal para promover narrativas intergeneracionales con el fin de "reconstruir opiniones individuales más apropiadamente" (Flick, 2004, p. 128). Además, permitió fortalecer un conocimiento propio que permita otorgarles un nuevo sentido a los adultos mayores que transformara sus experiencias.

- Técnica - Trabajo grupal e informativo: Se presentaron los dos instrumentos diseñados por medio de una mesa de trabajo y se mostró la forma en la que serían aplicados. Luego se hizo una rápida prueba piloto para validar los ajustes que recomendaron hacerle.

- Herramientas: Entrevista semiestructurada, taller reflexivo y mesa de trabajo.

- Insumos: Guía de Memoria 4 en la que se realizó el acta de la actividad.

Aplicación de los instrumentos para la recopilación de información - fuentes primarias

- Trabajo individual: Se perfeccionaron los instrumentos de acuerdo con el encuentro realizado en la actividad anterior y se diseñó un protocolo para su aplicación. Luego, se convocó una cita con el grupo de sistematización.

- Técnica - Trabajo grupal e informativo: Se aplicaron los dos instrumentos, siguiendo los protocolos establecidos.

- Herramientas: Entrevista semiestructurada y taller reflexivo. 
Cuello-Lacouture y Jaramillo-Jaramillo

- $\quad$ Insumos: Guía de Memoria 4, en la que se realizó el acta de la actividad.

Validación, interpretación y análisis de la información obtenida: taller de retroalimentación

- Trabajo individual: Durante dos semanas se organizó la información recolectada con base en los resultados relevantes por instrumento: descripción de datos, síntesis de los resultados ordenados por ejes y preguntas centrales problematizadoras desde la identificación de categorías y subcategorías de análisis, en función de los momentos de la experiencia.

Seguidamente, se preparó una presentación en diapositivas para socializar la información en un taller de retroalimentación.

- $\quad$ Técnica - Trabajo grupal e informativo: Socialización de resultados y realización de un taller grupal de retroalimentación.

- Herramientas: Taller de retroalimentación.

- Insumos: Guía de Memoria 4, en la que se realizó el acta de la actividad y grabadora de audio.

Plan de uso y comunicación

- Trabajo individual: Teniendo en cuenta que el Plan de uso y comunicación tiene el objetivo de definir cómo será usada y publicada la experiencia sistematizada, se determinó que la población objetivo para la comunicación son las dos familias participantes y los informantes calificados, entre los cuales están los vecinos y familiares que aportaron como testigos. Adicionalmente, se concretó que el medio de comunicación es una cartilla donde se expusieron específicamente los resultados obtenidos, los derechos del adulto mayor, las lecciones aprendidas y las conclusiones. Esta cartilla fue impresa a color y se entregó a cada uno de los participantes. Más adelante se contempló la posibilidad de visitar dos hogares geriátricos de las ciudades de Cali y Valledupar, con el fin de socializar la cartilla.

- Técnica - Trabajo grupal e informativo: Se convocó a las familias y a los informantes calificados para hacer entrega de la cartilla de manera personalizada.

- Herramienta: Taller de cierre.

- Insumos: Informe final, cartilla. 
Cuello-Lacouture y Jaramillo-Jaramillo

\section{Hallazgos}

El Proyecto de Asesoría Familiar titulado "Reconocimiento de los derechos humanos del adulto mayor en dos familias residentes en Cali y Valledupar" surge de la necesidad de intervenir una población que está incrementándose de manera progresiva, para contribuir a su empoderamiento a partir del reconocimiento de sus derechos humanos con el fin de incentivar su transformación a nivel individual, familiar y social.

A pesar de que en Colombia existe una normatividad orientada a la protección de los adultos mayores, su atención requiere de la participación de diversos sectores que circundan la población; por esta razón, se debe tomar conciencia y asumir la responsabilidad de promocionar los derechos humanos por medio de actividades que los involucren en primera medida a ellos, luego a sus familias como sistema de interacción y formación directa. Así mismo, la participación de otras redes como los vecinos y los amigos hace imprescindible la creación de una cultura del envejecimiento que valore y enaltezca esta etapa de la vida.

\subsection{Re-creación compartida de la experiencia y co-construcción de sentidos}

Los resultados acerca de la re-creación compartida de la experiencia y construcción de sentidos se presentan en dos perspectivas: una, desde la asesoría familiar a través de la perspectiva de las asesoras, y dos, desde los factores comunes y diferenciadores gestados en las experiencias de las familias S. P. y C. V., y terceros. Cada perspectiva recrea los momentos de la experiencia: (a) la situación inicial y su contexto: origen del proyecto de asesoría familiar; (b) el proceso de formulación del proyecto de asesoría familiar; (c) el proceso de implementación del proyecto de asesoría familiar; (d) la situación final: los resultados y sus efectos. Cabe anotar que la segunda perspectiva recrea estos momentos a partir de cada uno de los ejes definidos para la organización de la experiencia.

\subsection{Descripción y análisis de la experiencia desde la perspectiva de la asesoría familiar}

\subsubsection{La situación inicial y su contexto: Origen del Proyecto de Asesoría Familiar}

En un primer contacto con las familias, las asesoras desarrollaron un proceso de investigación acción participativa a través de un encuentro semanal, con dos familias ubicadas, una en la ciudad de Valledupar del departamento del Cesar y la otra en la ciudad de Cali del departamento del Valle del Cauca, durante el mes de febrero hasta el mes de noviembre del año 2017. Si bien, cada encuentro fue consensuado con ambas familias para que se realizara en su lugar de vivienda o en otro espacio, también, las familias se 
Cuello-Lacouture y Jaramillo-Jaramillo

comprometieron de manera voluntaria y responsable a participar en todos los encuentros desde el primer día hasta la finalización de estos. En concordancia, las familias rememoraron su pasado desde el instante en que comenzó su historia de amor, sus primeros trabajos, la llegada de los hijos, las enfermedades, las crisis económicas y las pérdidas, reconociendo su existencia y otorgando un mayor significado a su presente y a lo que esperan del futuro.

En ese sentido, se efectuó un diagnóstico social de la familia para identificar sus necesidades sociales desde sus testimonios, esto significa que el proceso se generó al interior de esta. Por tal razón, se aplicaron una serie de técnicas como el debate y la entrevista grupal semiestructurada.

Por otra parte, los encuentros constituyeron una experiencia profunda y significativa para la construcción de conceptos útiles que aportaron al Proyecto Asesoría Familiar y, además fue todo un desafío en el tiempo mantener a las familias motivadas. Incluso, se originaron inquietudes referentes al papel de lo social en la construcción del concepto de adultez mayor en la contemporaneidad, sobre todo, con relación a la concepción que en Occidente se tiene de esta población considerada como vulnerable, que en algunos casos invisibiliza y excluye a los adultos mayores.

A propósito, el desarrollo de los encuentros con las familias facilitó la creación y recreación de escenarios en los que confluyeron procesos de comunicación, relaciones y conexiones entre los diversos integrantes que están inscritos en un tiempo y espacio determinado. Al mismo tiempo, en el proceso de asesoría familiar se indagó y penetró en las diversas realidades planteadas por las familias, profundizando en sus historias de vida.

\subsubsection{El proceso de formulación del Proyecto de Asesoría Familiar}

El Proyecto de Asesoría Familiar convoca a dimensionar las familias desde una mirada multidimensional, transdisciplinaria e integradora. Además, como proceso de intervención es contextual y reflexivo, ya que implica reconocer las condiciones relacionales e idiosincráticas de las familias que emergen en los escenarios de acción.

De acuerdo con los planteamientos de Parra y Gutiérrez (2010), la principal característica de este proyecto fue que combinara de forma indisociable la investigación como proceso de indagación y producción de nuevos conocimientos con la intervención social que, busca aportar a la transformación de aspectos de la realidad concreta para reconocer a las familias escogidas como sujetos históricos, con una organización social y normativa, en la que se configuran identidades, particularidades y valores legitimados por sus integrantes. 
Cuello-Lacouture y Jaramillo-Jaramillo

Es así como, la intención como asesoras e investigadoras al comenzar a tener contacto con las familias, fue observar su interacción con el contexto, comprender el sentido que otorgan a su cotidianidad a través de sus historias, narrativas, lenguajes y experiencias, asumiendo el papel de observadoras que observan a observadores (Pakman, 1995). También en la acción, entender que se incursiona en un juego interaccional a nivel interno y externo, lo que implica como asesoras familiares unas prácticas reflexivas que según Morin y Pakman (1994), conduce a ver la realidad a través de los ojos de los demás y los de nosotras mismas: observar y auto-observar para lograr una reflexión dentro del proceso social.

Adicionalmente, la importancia del observado y del observador desde la acción de las asesoras al incorporarse a un sistema familiar ajeno, es reconocer la autonomía de cada integrante y su capacidad para transformar la sociedad, con relación al sistema de referencia relativo a la realidad (Foerster, 1991).

Es fundamental destacar que, los encuentros permitieron que las familias comprendieran sus dinámicas, las interacciones sociales y los discursos que se engendran en relación con otros. Igualmente, se vislumbró la comunicación como elemento principal de la interacción social y contribuyó en la construcción de significados de las experiencias individuales y colectivas que determinaron las acciones del entramado familiar. De esto se derivó la importancia del conocimiento y los significados articulados a creencias, ideologías y paradigmas que conllevan a acciones y prácticas sociales.

Particularmente, el Proyecto de Asesoría Familiar estuvo orientado a generar alternativas y estrategias para que los adultos mayores de estas familias se reconozcan como seres activos y vitales, que aportan a la sociedad y así, afianzar y consolidar sus vínculos afectivos entre el mismo grupo poblacional, con su familia y otras redes que los empodere y los transforme en copartícipes de su vida y en relación con los contextos de extensión.

\subsubsection{El proceso de implementación del Proyecto de asesoría familiar}

Los encuentros semanales con las familias iniciaron con una conversación comprendida como la convergencia de las distintas versiones que cada una tiene de su realidad, a lo largo de las conversaciones las familias definen y redefinen los significados de su experiencia como sujetos sociales en constante transformación y en relación con otros integrantes.

En las actividades desarrolladas durante el proceso de implementación, se gestaron experiencias compartidas con las familias y terceros, para facilitar la transformación dialógica que evoluciona en el tiempo y la consciencia ante los cambios perpetuados por la exteriorización de sentimientos y los pensamientos emergentes en cada espacio. Por esto, desde la asesoría familiar, el facilitar un espacio exclusivo de reflexión y diálogo expansivo 
Cuello-Lacouture y Jaramillo-Jaramillo

en familia, abre el camino para que se logre una comprensión profunda de los derechos humanos, reconociendo la dimensión sistémica del ser humano relacional como un todo, que construye su historia y busca transformar la sociedad, a través del conocimiento generado desde sus experiencias.

En la implementación práctica de la asesoría familiar con adultos mayores se destacó el valor de las relaciones intergeneracionales como proceso bidireccional que promueve el intercambio de conocimientos y la cooperación entre las familias, para originar una nueva cultura de vejez y envejecimiento que favorezca la participación social de esta población como sujeto de derechos. Así mismo, en los escenarios intergeneracionales emerge una cultura de transmisión de experiencias a partir de los diálogos que se dan entre abuelos y nietos. Además, es significativa la actuación que desempeña la familia, el Estado y la sociedad como corresponsables del cuidado al adulto mayor, puesto que, legislativamente son garantes de sus derechos.

Para concluir, se puede afiarmar que el ser humano en su multidimensionalidad y complejidad necesita el contacto con los otros para desarrollarse integralmente y aprender sobre el mundo. Es así como esa interacción está determinada por pautas comunicacionales que otorgan significado a las experiencias: "las personas dan sentido a sus vidas y relaciones relatando su experiencia y que, al interactuar con otros en la representación de estos relatos, modelan sus propias vidas y relaciones" (White y Epston, 1993, p. 30).

\subsubsection{La situación final: los resultados y sus efectos}

En el momento final se generaron cambios correspondientes a cada uno de los ejes como resultado de ambas experiencias. En términos generales, la asesoría familiar posibilitó dimensionar a las familias como unidad visible de interconexiones vinculares con un carácter dinámico propio que se reconoce en la convergencia de las interdependencias en constante transformación e interacción recíproca con el contexto. Por tal razón, valoramos a las familias desde su contexto sociocultural de manera flexible y compleja para construir y resignificar sus experiencias, que se moldean en costumbres, comportamientos, actitudes, normas, valores y demás; en ese sentido, la familia constituye un contexto fundamental para la construcción del desarrollo individual, social e intergeneracional.

$\mathrm{Al}$ respecto, como asesoras familiares resignificamos las pautas comunicacionales que determinan la manera de ser de cada integrante de la familia y de terceros, también, la forma de expresarse ante los demás y redefinirse. Es por esto que se reconoce la importancia del lenguaje en los seres humanos, puesto que somos seres particularmente lingüísticos y no hay lugar fuera del lenguaje en el que podamos observar y describir nuestra existencia individual y social. 
Cuello-Lacouture y Jaramillo-Jaramillo

De cierto modo, el lenguaje es la regulación funcional del comportamiento y los seres humanos poseen la capacidad para comunicarse de forma reflexiva con ellos mismos y con otros, fundamentando la razón humana (Echeverría, 1994). Así mismo, en la etapa de la vejez se produce una introspección natural en la que los adultos mayores se transforman en observadores de su propia realidad, se comprende el significado de la vida y se acentúa el deseo de apoyar a otros aumentando su generatividad (Erikson, 2000).

A propósito, desde la asesoría familiar se reconoce a la población de adultos mayores como sujeto de derechos, capaces de influir de forma directa en la sociedad suscitando la preservación de las tradiciones, los valores y los legados culturales: “(...) los abuelos son la sabiduría de la familia, son la sabiduría de un pueblo. Y un pueblo que no escucha a los abuelos es un pueblo que muere" (Bergoglio, 2013).

Se comprende que los adultos mayores lograron percibirse como receptores de apoyo y proveedores de este en extensión a sus redes, además son conscientes del protagonismo que desempeñan las relaciones intergeneracionales al interior de la familia, la comunidad y la sociedad para el empoderamiento de sus derechos. Así mismo, la familia es un sistema en constante desarrollo, "no es el individuo el que (con la familia al fondo) va atravesando una serie de etapas en el transcurso del tiempo, sino la familia como tal la que evoluciona" (Friedman, 1989). Por tal razón para reconocer a las familias como sujetos portadores de cultura, debemos comprender los diferentes contextos sociales de influencia. No obstante, las familias y terceros se asumieron desde la asesoría familiar como sujetos colectivos de derechos y agentes garantes de estos.

\section{Conclusiones}

Pensarse en la población de adultos mayores, requiere considerar la diversidad inherente a la naturaleza humana, donde la cultura se torna transversal en las dinámicas y las transformaciones individuales, familiares y colectivas en sociedad. En ese sentido, la vejez es un proceso significativo en cada historia de vida; es por lo que se debe abordar como plantea Minuchin (2009) desde la estructura y la organización de las experiencias de los mismos integrantes de la familia. Por consiguiente, la participación de las familias como sistema durante todo el proyecto de asesoría familiar y sistematización de la experiencia fue importante para co-construir canales donde el saber fluyera a través de las narrativas, se deconstruyeran las realidades dominantes para conceder paso a resignificaciones de lo que representa ser un adulto mayor sujeto de derechos.

Es evidente que los adultos mayores y sus familias en la etapa inicial del proyecto de asesoría familiar vivían inmersos en sus dinámicas cotidianas prescindiendo de la generación de elementos reflexivos frente a la misma. Sin embargo, en los procesos de implementación y resultados, se identificó una clara consciencia de los integrantes de las 
Cuello-Lacouture y Jaramillo-Jaramillo

familias sobre el rol del adulto mayor como sujeto de derechos humanos. Por esta razón, el adulto mayor logró experimentarse como un sujeto de derechos en la medida que participó en sus diferentes escenarios de vida: familiar, social y religioso.

Por cierto, desde la perspectiva sistémica, los aportes obtenidos durante los procesos de aprendizajes colectivos facilitaron el fortalecimiento de los vínculos intergeneracionales, el protagonismo social y el empoderamiento de los adultos mayores como sujetos de derechos portadores de un legado histórico de vida. Cabe resaltar que los encuentros intergeneracionales fueron esenciales para generar un intercambio dialéctico entre los sistemas, en los que emergieron reestructuraciones de los roles que contribuyeron a un cambio en las familias y terceros, por medio de una mayor integración afectiva, inclusión y participación social en la cotidianidad, cumpliendo el objetivo de coadyuvar al empoderamiento de los adultos mayores como sujeto de derechos.

Al respecto, Galvis (2009) afirma que la participación es un derecho fundamental que se tiene de ser partícipes activos en los procesos de elaboración y de gestión de proyectos, como se desarrolló con las familias S. P. en Valledupar y C. V. en Cali.

Además de la participación, el ejercicio de los derechos en el adulto mayor también es determinado por las acciones de corresponsabilidad dentro del sistema familiar y social. La familia, la sociedad y el Estado son agentes corresponsables del cuidado, protección y apoyo a los adultos mayores a través de acciones que propendan por el bienestar de esta población considerada como vulnerable. En consecuencia, todos somos garantes de su calidad de vida y se debe promover desde contextos más cercanos, como el vecindario, la iglesia y las Juntas de Acción Comunal. Es así como los adultos mayores son beneficiarios de apoyo y agentes de este, en expansión a sus redes, siendo conscientes del protagonismo social y las relaciones intergeneracionales al interior de la familia, la comunidad y la sociedad para el fortalecimiento de sus derechos humanos. De esta manera, desde la asesoría familiar se concibe a la familia como un sistema que transita por diversas etapas y evoluciona en el tiempo para reconocerse como portadora de cultura en los diferentes contextos que la permean.

En efecto, revelar desde perspectivas teóricas y prácticas los principales logros del proceso de formulación e implementación del proyecto de Asesoría Familiar, como otro de los objetivos, permitió concebir la vejez como una etapa del ciclo vital en la que se cumple el rol de agentes de conocimiento para las generaciones descendientes, puesto que son el resultado de todas las experiencias y las transformaciones que se gestaron en etapas anteriores. Entonces, el envejecimiento es planteado como un proceso que se vive de forma individual en el que confluyen diversos factores psicosociales y contextuales que determinan la calidad de vida de la población. 
Cuello-Lacouture y Jaramillo-Jaramillo

Valorar el ejercicio de los derechos del adulto mayor desde una perspectiva sistémica, convoca también a apreciar la importancia de contar con redes interinstitucionales de apoyo, que contribuyan como soporte para estimular la participación de los mayores en espacios distintos a los familiares. En ese orden de ideas, la familia con todo su potencial a través de cada integrante es quien puede hacer de esta última etapa del ciclo vital una experiencia maravillosa en donde la cooperación, la ayuda, el estímulo, el reconocimiento, la creatividad y la valoración de sus mayores permita el crecimiento y el afianzamiento de los valores aprendidos, el enriquecimiento a partir de las vivencias cotidianas para alcanzar una vejez digna.

Con lo precedente, se contribuye a cambiar los paradigmas sociales existentes con relación a los adultos mayores y sus posibles limitaciones, promulgando sus experiencias por medio de campañas permanentes que generen expectativas y resalten sus habilidades.

Para finalizar, la conjugación de los procesos de investigación y asesoría familiar fueron determinantes y contribuyeron en la transformación de realidades puntuales dentro de las familias y los terceros, ya que se incentivó la participación social y la corresponsabilidad para el empoderamiento de los derechos humanos del adulto mayor.

\section{Referencias Bibliográficas}

Alboan, Hegoa, y Universidad de Deusto. (2004). La sistematización, una nueva mirada a nuestras prácticas. Guía para la sistematización de experiencias de transformación social. Bilbao: Alboan; Hegoa; Universidad de Deusto. Recuperado de http://publicaciones.hegoa.ehu.es/uploads/pdfs/64/Guia_Sistematizaci_n_2004. pdf?1488539204.

Ayllón, M. (octubre, 2001). La práctica como fuente de conocimiento: Una propuesta operativa para sistematizar experiencias en trabajo social. En XVII Seminario Latinoamericano de Escuelas Trabajo Social. Pontificia Universidad Católica del Perú, Lima, Perú.

Barnechea, M., y Morgan, M. (2007). El conocimiento desde la práctica y una propuesta de método de sistematización de experiencias (Tesis de maestría). Pontificia Universidad Católica del Perú. Lima, Perú.

Bazo, M., y García, B. (2006). Envejecimiento y sociedad: Una perspectiva Internacional. (2 ed.). Madrid, España: Editorial Panamericana.

Bergoglio, J. (2013). Siete frases papa francisco dedicadas abuelos ancianos. Divina Misericordia. Recuperado de https://elmisericordioso.me/7-frases-del-papafrancisco-dedicadas-a-los-abuelos.

Carvajal-Burbano, A. (2006). Teoría y práctica de la sistematización de experiencias. Cali: Universidad del Valle. Facultad de Humanidades. Escuela de Trabajo Social y Desarrollo Humano. 
Cuello-Lacouture y Jaramillo-Jaramillo

Cotera-Fretel, F. (2012). Manual: elaboración de proyectos de desarrollo. Dirigido a integrantes de instituciones promotoras de desarrollo y organizaciones sociales. Lima, Perú: Comunicaciones aliadas.

Departamento Administrativo Nacional de Estadística. (2017). Proyecciones nacionales y departamentales de población 2005-2020. Bogotá: Centro Andino de Altos Estudios (CANDANE).

Echeverría, R. (1994). Ontología del lenguaje. Chile: Comunicaciones Noreste Ltda.

Elías, N. (2009). La soledad de los moribundos. México: Editorial fondo de cultura económica.

Erikson, E. (2000). El ciclo vital completado. Barcelona, España: Paidós Ibérica.

Flick, U. (2004). Introducción a la investigación cualitativa. Madrid: Morata.

Foerster, H. (1991). Las semillas de la cibernética: obras escogidas. Barcelona: Gedisa

Friedman, E. H. (1989). Systems and ceremonies: a family view of rites of passage. En B. Carter y M. McGoldrick (Eds), The changings family life cycle. A framework for family therapy (pp. 335-347). New York: Allyn and Bacon.

Galvis, L. (2009). La Convención de los Derechos del niño veinte años después. Revista Latinoamericana de Ciencias Sociales, Niñez y Juventud, 2(7), 587-619.

Ghiso, A. (agosto, 1999). De la práctica singular al diálogo con lo plural. Aproximaciones a otros tránsitos y sentidos de la sistematización en épocas de globalización. En Seminario Latinoamericano: Sistematización de Prácticas de Animación Sociocultural y Participación Ciudadana en América Latina. Universidad Católica Luis Amigó, Medellín, Colombia.

Minuchin, S. (2009). Familias y terapia familiar. Barcelona, España: Editorial Gedisa.

Morin, E., y Pakman, M. (1994). Introducción al pensamiento complejo. Barcelona: Gedisa.

Pakman, M. (1995). Investigación e intervención en grupos familiares: Una perspectiva constructivista. En J. M. Delgado y J. Gutiérrez, Métodos y técnicas cualitativas de investigación en ciencias sociales (pp. 359-377). Madrid, España: Síntesis.

Parra, M., y Gutiérrez, P. (2010). Encuentro 4: ¿Qué es intervenir? ¿Qué es investigar? Algunas reflexiones en torno a procesos de intervención/investigación en contextos comunitarios y de movilización social. Recuperado de http://proyectos.javerianacali.edu.co/cursos_virtuales/posgrado/maestria_asesori a_familiar/proyectos_I/modulo1/unidad1/Parra_Intervenci\%C3\%B3n\%20Investiga ci\%C3\%B3n_.pdf.

Torres-Carrillo, A. (octubre, 1996). La sistematización como investigación interpretativa crítica: Entre la teoría y la práctica. En Seminario Internacional sobre Sistematización y Producción de Conocimiento para la Acción. CEAAL, Santiago, Chile.

White, M., y Epston, D. (1993). Medios narrativos para fines terapéuticos. Barcelona: Paidós. 


\section{OTROS ARTÍCULOS DE PROSPECTIVA No. 31 DE 2021}

\section{PRESENTACIÓN}

Presentación. Reflexiones sobre desafios al publicar sistematizaciones

Rosa María Cifuentes-Gil

\section{EDITORIAL}

Reflexiones sobre Trabajo Social: aportes de la Sistematización

María Rocío Cifuentes-Patiño

\section{ARTÍCULOS}

Hacer lo que se sabe, pensar lo que se hace. La sistematización como modalidad investigativa Alfonso Torres-Carrillo

Aportes y desafios de la Sistematización de experiencias en el Trabajo Social y la extensión crítica. Apuntes y reflexiones desde la perspectiva de la Educación Popular

María Rosa Goldar

Valeria Chiavetta

La sistematización en Trabajo Social y la epistemología feminista del punto de vista. Diálogos sobre la producción de conocimiento sustentada en experiencias

Ruth Noemí Parola

María Florencia Linardelli

La Sistematización investigativa de las experiencias: del baile de los que sobran a la fiesta de los que faltan

María Belén Ortega-Senet

Sistematización y Trabajo Social en Chile. El largo y sinuoso camino

Patricia Lorena Castañeda-Meneses

Ana María Salamé-Coulon
Sentipensar la pandemia COVID-19 desde la sistematización de la experiencia en Trabajo Social: reflexiones del profesor Oscar Jara Holliday

Elia Sepúlveda-Hernández

La sistematización de experiencias, una investigación social cualitativa que potencia buenas prácticas de convivencia y gobierno. La experiencia de un conjunto residencial multifamiliar en Cali, Colombia Martha Lucia Echeverry-Velásquez Manuela Prada-Dávila

Construcción de subjetividades epistemológicaspolíticas de profesoras y profesores de Investigación social en una universidad privada y confesional en Bogotá

Giovanni Mora-Lemus

Sistematización de la experiencia Reconocimiento de los derechos humanos del adulto mayor en dos familias residentes en Cali y Valledupar (Colombia)

Lina María Cuello-Lacouture

Jimena del Pilar Jaramillo-Jaramillo

La memoria transformadora como estrategia de intervención profesional en los procesos de reconciliación social: comprensión a partir de mujeres campesinas, excombatientes y jóvenes en Manizales, Colombia

Yeimmy Stephania Corredor-Sotelo

Juliana Fuertes-Fuertes

Sistematización de una estrategia de educación informal implementada en personas privadas de la libertad en el establecimiento penitenciario de mediana seguridad y carcelario de Barranquilla, Colombia

Rafael Humberto Herrera-Mercado Rafael Alberto Zambrano-Vanegas 
Aportes significativos del proceso de intervención comunitaria con la Escuela Popular de Comunicación Alternativa Jaime Garzón de la ciudad de Cúcuta, Colombia

Carlos Lasso-Urbano

La sistematización de la intervención como metodología de investigación en Trabajo Social. Importancia práctica y teórica de la fase de recogida de datos en la intervención social según experiencia del Programa de Apoyo a las Familias en Zaragoza, España

Elisa Esteban-Carbonell

Nuria Del Olmo-Vicén

Papel de la sistematización de experiencias en los procesos de evaluación de intervenciones de salud pública en la Comuna Saludable por la Paz, Cali - Colombia

Jenny Faisury Peña-Varón

Paola Andrea Marín-Velásquez

Janeth Mosquera-Becerra

Experiencia de intervención social en hogares comunitarios integrales del barrio Alfonso Bonilla Aragón, Cali - Colombia

Julián Alexander Montaño-Cárdenas

Las políticas sociales y el gobierno de la "población indígena". Estrategias y regulaciones en el multiculturalismo chileno

Rodrigo Agustín Navarrete-Saavedra

Representaciones sociales sobre estilo de autoridad y tipos de interacción en cuidadores de residencias de protección infantil en Chile

Marcelo Gallegos-Fuentes
Carmen Gloria Jarpa-Arriagada

Reflexiones sobre inseguridad social y cuestiones penales. Una respuesta estratégica a partir de experiencias de cooperativismo con ex detenidos en Argentina

Analia Elizabeth Otero

Yael Yanina Barrera

Desarrollo y salud: la emergencia de un nuevo paradigma

Jesús María Sánchez-Ordóñez

Trabajo Social en ejercicio libre: la perspectiva profesional en España

Paula Frieiro-Padín

Tamara Fernández-Arias

Rubén González-Rodríguez

\section{RESEÑAS}

Social Work and the City: Urban Themes in 21stCentury Social Work

Felipe Saravia-Cortés

Respuestas del Trabajo Social ante emergencias sociales y problemáticas sociales complejas de México y España

Felipe Saravia-Cortés

El feminismo, el género y la profesionalización del trabajo social en Colombia (1936-2004)

Ambar Oriana Serna-Lombo

El puño invisible. Arte, revolución y un siglo de cambios culturales

Carlos Arturo Robledo-Marín

\section{PROSPECTIVA}

No. $31 \bullet$ ene.-jun. 2021

e-ISSN: 2389-993X • Universidad del Valle 